\title{
Drug Release Profile from Calcium-Induced Alginate-Phosphate Composite Gel Beads
}

\author{
Yoshifumi Murata, Youko Kodama, Takashi Isobe, Kyoko Kofuji, and Susumu Kawashima
}

Faculty of Pharmaceutical Science, Hokuriku University, Ho-3 Kanagawa-machi, Kanazawa, Ishikawa 920-1181, Japan

Correspondence should be addressed to Yoshifumi Murata, y-murata@hokuriku-u.ac.jp

Received 10 September 2008; Accepted 20 November 2008

Recommended by Sung Chul Kim

\begin{abstract}
Calcium-induced alginate-phosphate composite gel beads were prepared, and model drug release profiles were investigated in vitro. The formation of calcium phosphate in the alginate gel matrix was observed and did not affect the rheological properties of the hydrogel beads. X-ray diffraction patterns showed that the calcium phosphate does not exist in crystalline form in the matrix. The initial release amount and release rate of a water-soluble drug, diclofenac, from the alginate gel beads could be controlled by modifying the composition of the matrix with calcium phosphate. In contrast, the release profile was not affected by the modification for hydrocortisone, a drug only slightly soluble in water.
\end{abstract}

Copyright (C) 2009 Yoshifumi Murata et al. This is an open access article distributed under the Creative Commons Attribution License, which permits unrestricted use, distribution, and reproduction in any medium, provided the original work is properly cited.

\section{Introduction}

The calcium salt of phosphoric acid (CP) is distributed widely in nature; several types of $\mathrm{CP}$ are constituents of animals. For example, hydroxyapatite is a component of bones and teeth in humans. In general, CP is formed by the coexistence of phosphate ions with calcium ions. CP is a harmless material and is used as an additive in foods and medicines; for example, as an active ingredient to protect against dental caries. The suitability of $\mathrm{CP}$ as an injectable material has been investigated because of its biocompatibility $[1,2]$, and CP-polymer composites have been studied as functional biomaterials [3-7]. Natural polysaccharides, which have also been used widely in the food industry, serve as dietary fiber and are not absorbed in the gastrointestinal tract. An anionic polysaccharide, alginic acid, has been used as a medicine for stomach ulcers as well as a food additive because of its protective effect on the gastric mucosa when given orally. Alginic acid undergoes a sol-gel transformation in response to cations; for example, a solution of sodium alginate immediately forms a cured gel matrix (Alg-Ca) in the presence of calcium ions. The Alg-Ca matrix is used not only as a vehicle for drug delivery, but also as a material in biomedical engineering [8-10].

An investigation of drug release profiles of $\mathrm{Alg}$-Ca showed that the release rate could be controlled by coating and modifying the composition of the gel matrix [1113]. However, the controlled release of a water-soluble drug from Alg-Ca is difficult because the drug immediately diffuses through the matrix to the gel surface. Therefore, a modification of the gel matrix structure is necessary to control drug release from the Alg-Ca [14]. In the present study, calcium alginate-CP composite gel beads (CAPs) containing a drug were prepared by a simple method in aqueous solution and the rheological properties of CAP were tested, followed by the investigation of the drug release profile from dried CAP in vitro.

\section{Materials and Methods}

2.1. Materials. Sodium alginate and diammonium hydrogen phosphate (AP) were obtained from Nacalai Tesque, Inc. (Kyoto, Japan; $300 \mathrm{cps}$ ). Sodium diclofenac (DF), hydrocortisone (HC), $\mathrm{Ca}\left(\mathrm{H}_{2} \mathrm{PO}_{4}\right)_{2}, \mathrm{CaHPO}_{4}, \mathrm{Ca}_{3}\left(\mathrm{PO}_{4}\right)_{2}$, and hydroxyapatite (particle size $46-149 \mu \mathrm{m}$ ) were purchased from Wako Pure Chemical Industries (Osaka, Japan). All other reagents used were of analytical grade.

2.2. Preparation of $C P$ Powder. $C P$ was prepared by the reaction between calcium and ammonium phosphate in aqueous solution as follows. Equal volumes of $8 \% \mathrm{AP}$ and $9.5 \% \mathrm{CaCl}_{2}$ were mixed, resulting in the immediate 


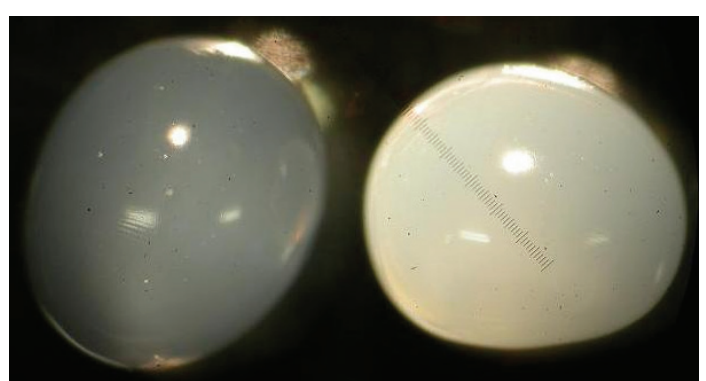

Figure 1: Pictures of Alg-Ca (left) and CAP (right) prepared with $0.8 \%$ AP.

appearance of a white precipitate. The suspension was stirred with a magnetic stirrer for one day at room temperature and then centrifuged at $3000 \mathrm{rpm}$ for 5 minutes. The precipitate was collected and washed three times with water, then dried on a dish. It was desiccated under vacuum in the presence of $\mathrm{P}_{2} \mathrm{O}_{5}$.

2.3. Preparation of CAP. CAP was prepared as follows: $1.5(\mathrm{w} / \mathrm{w}) \%$ or $2.0(\mathrm{w} / \mathrm{w}) \%$ sodium alginate solution was prepared with demineralized water and AP was added to the solution with agitation. The model drug was then added to $10 \mathrm{~g}$ of solution and mixed until homogeneity. Two grams of this solution then combined with $10 \mathrm{~mL}$ of $0.1-0.4 \mathrm{M} \mathrm{CaCl}_{2}$ solution and left to stand for 1 hour at room temperature, after which spherical hydrogel beads were obtained. The beads were washed twice with $50 \mathrm{~mL}$ distilled water and dried at $37^{\circ} \mathrm{C}$ for 24 hours on a culture dish, and then desiccated under vacuum in the presence of $\mathrm{P}_{2} \mathrm{O}_{5}$. The morphology of Alg-Ca was observed with a digital microscope (VHX200, Keyence Co., Osaka, Japan). The dried Alg-Ca or CAP prepared as described above was ground by electromotive mill (WB-1, Osaka Chemical Co., Osaka, Japan), and a sample of fine powder was used for X-ray diffractometry.

2.4. X-Ray Diffractometry. Powder X-ray diffractometry was conducted using an automatic diffractometer $\left(\mathrm{MXP}^{3}\right.$; MAC Science Ltd., Yokohama, Japan) with a voltage of $40 \mathrm{kV}$ and a current of $20 \mathrm{~mA}$. The scanning rate was $2^{\circ} \mathrm{min}^{-1}$ over a 2 theta range of $5-60^{\circ}$. The results of X-ray diffraction were interpreted using the XPRESS computer program (Bruker AXS K.K., Yokohama, Japan).

2.5. Rheological Test of Hydrogel Beads. The load weight (g) was measured three times with a rheometer (Rheo Tex, Sun Scientific Co. Ltd., Tokyo, Japan) at room temperature and a strain of hydrogel beads pressed with a flat adapter (diameter: $15 \mathrm{~mm}$ ) reached $0.5 \mathrm{~mm}$.

2.6. Drug Release Test from CAP. The release profile of the drug incorporated in CAP was determined in $500 \mathrm{~mL}$ of physiological saline using a JP XIII dissolution test apparatus (Toyama Sangyo Co., Tokyo; paddle method, $\left.37 \pm 0.5^{\circ} \mathrm{C}, 150 \mathrm{rpm}\right)$. A $5 \mathrm{~mL}$ aliquot of test solution was removed periodically and $5 \mathrm{~mL}$ of new medium $\left(37^{\circ} \mathrm{C}\right)$ were added to maintain a constant volume. Absorbance was

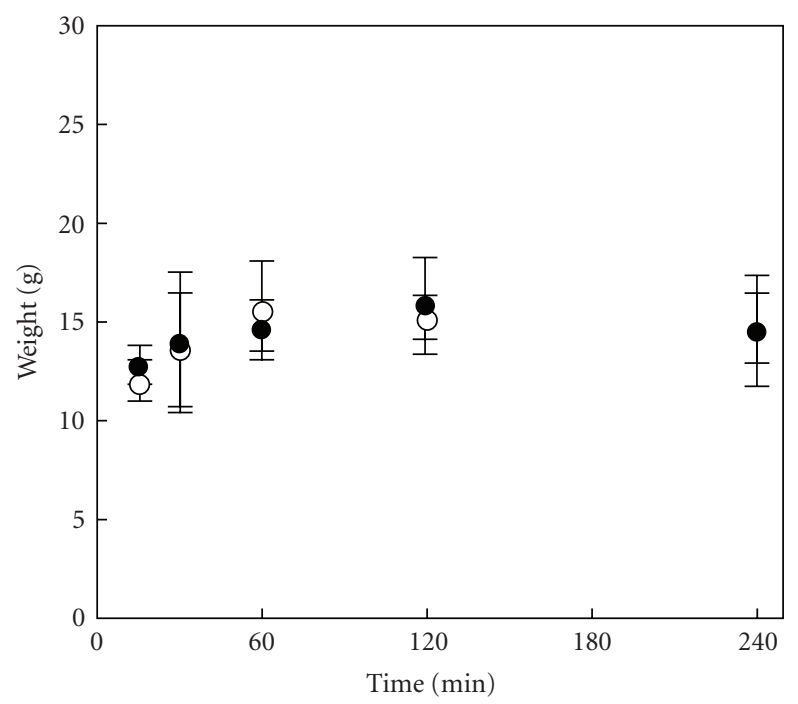

FIgURE 2: Change in hardness of hydrogel beads (mean \pm S.D., $n=$ 5). Open circles: Alg-Ca; closed circles: CAP (0.8\% AP).

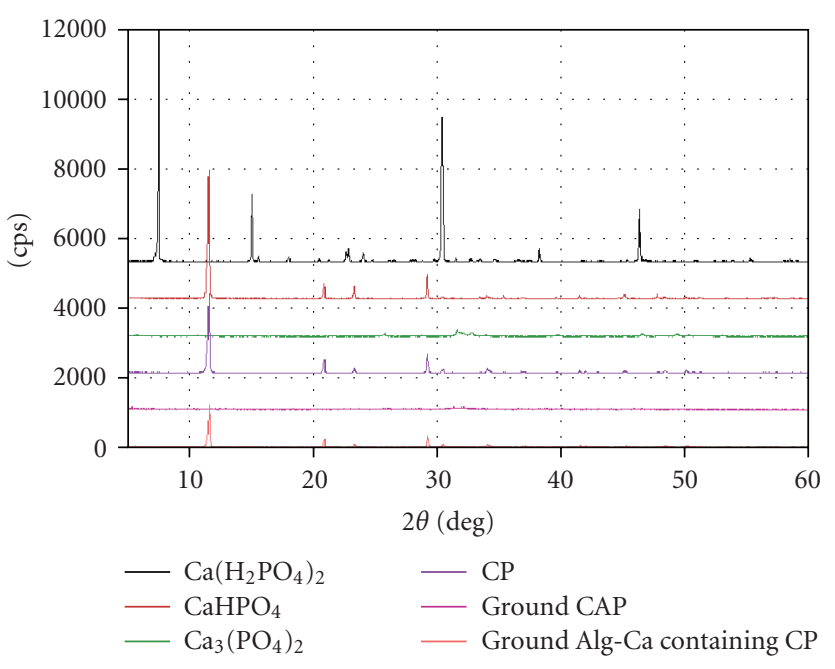

Figure 3: X-ray diffractograms. (1) $\mathrm{Ca}\left(\mathrm{H}_{2} \mathrm{PO}_{4}\right)_{2}$ (2) $\mathrm{CaHPO}_{4}$ (3) $\mathrm{Ca}_{3}\left(\mathrm{PO}_{4}\right)_{2}$ (4) CP (5) ground CAP (6) ground Alg-Ca containing $\mathrm{CP}$.

measured at $275 \mathrm{~nm}$ (for DF) or $248 \mathrm{~nm}$ (for HC) using an ultraviolet spectrometer (UV-1200, Shimadzu, Kyoto, Japan). All dissolution tests were performed in triplicate.

\section{Results and Discussion}

When $1.5 \%$ sodium alginate containing $0.8 \%$ AP was dropped into $0.2 \mathrm{M} \mathrm{CaCl} 2$ solution, a cured gel bead approximately $3.5 \mathrm{~mm}$ in diameter was formed. As shown in Figure 1, the formation of CP in alginate gel matrix was observed visually. A change in Alg-Ca also was observed when another phosphate, such as $\mathrm{Na}_{2} \mathrm{HPO}_{4}$ or $\mathrm{K}_{2} \mathrm{HPO}_{4}$, was added to the alginate solution. For $1.6 \%$ AP addition, aggregation of the gel beads occurred in the solution. The 
TABLE 1: Release parameters of DF from Alg-Ca or CAP prepared with $0.8 \%$ AP.

\begin{tabular}{lcccc}
\hline Sample & $\begin{array}{c}\mathrm{CaCl}_{2} \\
(\mathrm{M})\end{array}$ & $\begin{array}{c}\text { Release* } \\
(\%)\end{array}$ & $\begin{array}{c}\text { Release rate** } \\
\left(\% \min ^{-1}\right)\end{array}$ & $\begin{array}{c}\text { Incorporation } \\
(\%)\end{array}$ \\
\hline Alg-Ca & 0.1 & $19.0 \pm 2.1$ & $0.94 \pm 0.01$ & 68 \\
Alg-Ca & 0.2 & $18.9 \pm 1.9$ & $0.65 \pm 0.05$ & 88 \\
Alg-Ca & 0.4 & $16.8 \pm 1.8$ & $0.59 \pm 0.02$ & 90 \\
CAP & 0.1 & $4.0 \pm 0.2$ & $0.78 \pm 0.01$ & 74 \\
CAP & 0.2 & $1.2 \pm 0.1$ & $0.29 \pm 0.00$ & 95 \\
CAP & 0.4 & $1.0 \pm 0.2$ & $0.19 \pm 0.01$ & 94 \\
\hline
\end{tabular}

* Measured at 10 minutes, ${ }^{* *}$ calculated at $10-60$ minutes. Each release and release rate value includes mean \pm S.D. $(n=3)$.

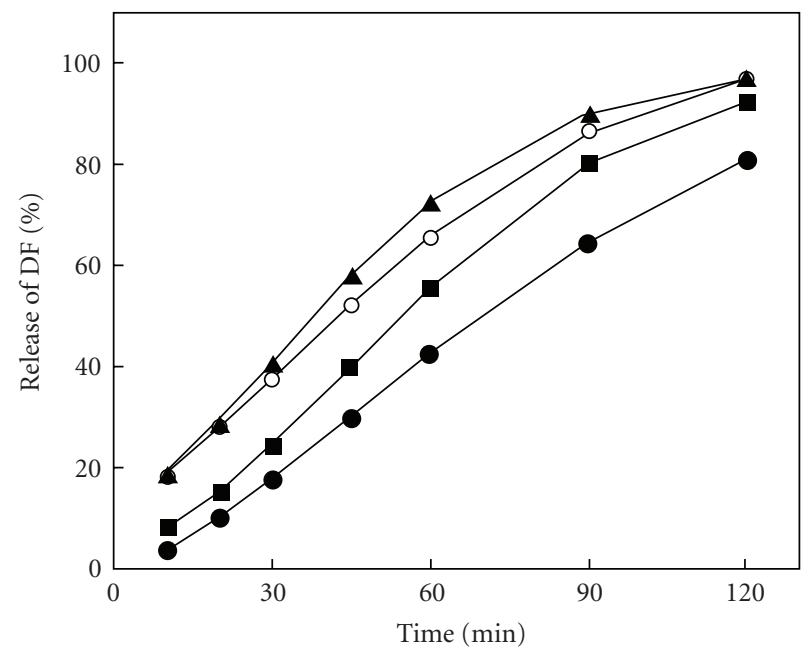

(a)

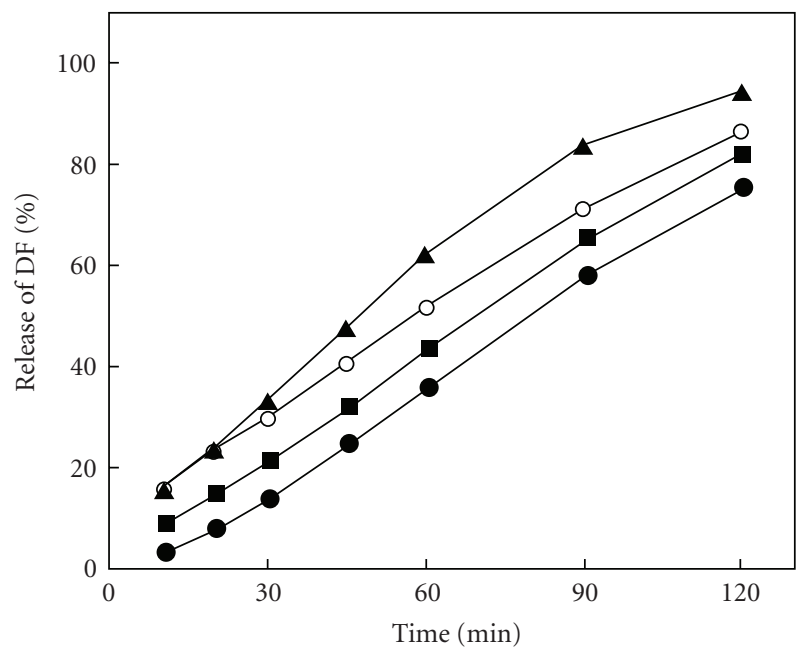

(b)

Figure 4: Release profiles of DF in physiological saline. (a) Prepared with $1.5 \%$ alginate and $0.1 \mathrm{M} \mathrm{CaCl}_{2}$. (b) Prepared with $2.0 \%$ alginate and $0.1 \mathrm{M} \mathrm{CaCl}_{2}$. Open circles: Alg-Ca; closed triangles: CAP (0.2\% AP); closed squares: CAP (0.5\% AP); closed circles: CAP (0.8\% AP).

results show that formation of calcium-induced alginate gel matrix and that of $\mathrm{CP}$ progressed simultaneously.

Figure 2 shows the mass (g) required for deformation of the hydrogel bead. The beads quickly stiffened in $\mathrm{CaCl}_{2}$ solution and a consistent hardness was achieved at about 1 hour. Addition of $0.8 \% \mathrm{AP}$ did not affect the rheological properties of Alg-Ca prepared with $1.5 \%$ alginate, and the CAP showed same profile as that obtained from Alg-Ca.

Figure 3 shows the X-ray diffraction data of $\mathrm{CPs}$ and the powder prepared by grinding dried Alg-Ca. Three species of calcium phosphate, $\mathrm{Ca}\left(\mathrm{H}_{2} \mathrm{PO}_{4}\right)_{2}, \mathrm{CaHPO}_{4}$, and $\mathrm{Ca}_{3}\left(\mathrm{PO}_{4}\right)_{2}$, each showed a different diffraction pattern. $\mathrm{CP}$ obtained from mixing the AP solution with a $\mathrm{CaCl}_{2}$ solution produced several X-ray diffraction peaks with a pattern compatible with $\mathrm{CaHPO}_{4}$. In addition, the powder prepared by grinding Alg-Ca containing $\mathrm{CP}$ also showed the same $\mathrm{X}$ ray diffraction pattern as $\mathrm{CaHPO}_{4}$. The crystal structure of $\mathrm{CP}$ was maintained even after Alg-Ca (hydrogel) containing $\mathrm{CP}$ was dried and ground using an electromotive mill. However, the diffraction pattern of ground CAP resembled that of ground Alg-Ca (free CAP), which produced a "halo" pattern. These results indicate that $\mathrm{CP}$ does not exist in a crystalline form in CAP.
Figure 4 shows the release profile of DF from dried AlgCa or CAP. Approximately 20\% of DF incorporated in AlgCa prepared with $1.5 \%$ alginate was released at 10 minutes. The initial release decreased according to the increment of AP added to the sodium alginate for the preparation of CAP; for example, release was $4.0 \pm 0.2 \%$ at 10 minutes for CAP prepared with $1.5 \%$ alginate containing $0.8 \%$ AP. However, the DF release profile from Alg-Ca containing 0.8\% hydroxyapatite was the same as that of Alg-Ca (data not shown). Thus, $\mathrm{CP}$ formed in Alg-Ca was assumed to act as a part of the dried gel matrix. A change in release rate was not clearly recognized unless $\mathrm{CaCl}_{2}$ concentration did not change. When Alg-Ca was prepared with $2.0 \%$ alginate, similar results were obtained.

DF is a water-soluble drug and is released immediately through routes formed by permeation of the dissolution medium into the dried alginate gel matrix. Calcium ions act as a raw material for formation of the alginate gel matrix, $\mathrm{CP}$, and/or alginate-phosphate complex.

Table 1 shows the release parameters of DF from Alg$\mathrm{Ca}$ or CAP prepared at various $\mathrm{CaCl}_{2}$ concentrations. For Alg-Ca (CP free), the amount of DF incorporated increased, and the release rate decreased, based on $\mathrm{CaCl}_{2}$ concentration 


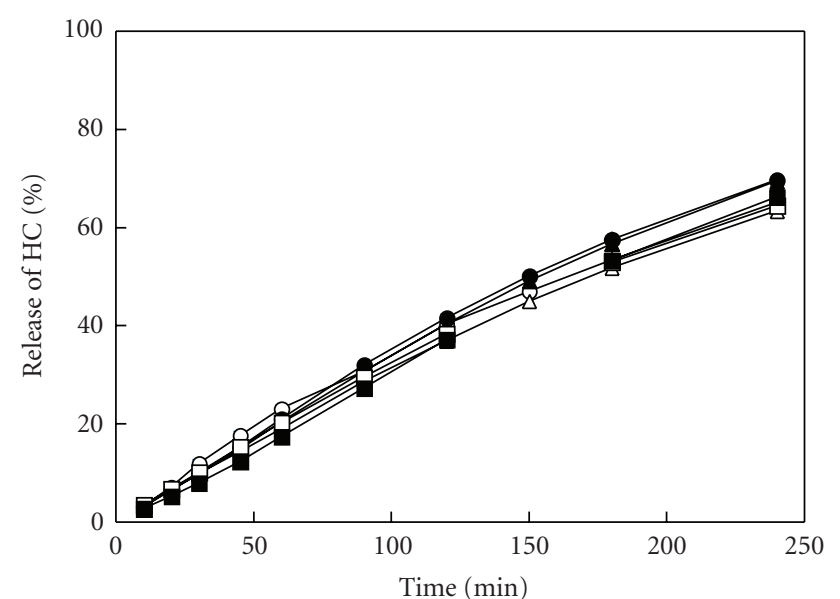

Figure 5: Release profiles of HC from Alg-Ca or CAP in physiological saline. Open circles: $\mathrm{Alg}-\mathrm{Ca}\left(1.5 \% \mathrm{Alg}, 0.1 \mathrm{M} \mathrm{CaCl}_{2}\right)$; open squares: Alg-Ca (1.5\% Alg, $\left.0.2 \mathrm{M} \mathrm{CaCl}_{2}\right)$; open triangles: $\mathrm{Alg}-\mathrm{Ca}$ (2.0\% Alg, $0.1 \mathrm{M} \mathrm{CaCl}_{2}$ ); closed circles: CAP (1.5\% Alg, 0.5\% AP, $0.1 \mathrm{M} \mathrm{CaCl}_{2}$ ); closed squares: CAP (1.5\% Alg, 0.8\% AP, $0.2 \mathrm{M}$ $\left.\mathrm{CaCl}_{2}\right)$; closed triangles: CAP $\left(2.0 \% \mathrm{Alg}, 0.5 \% \mathrm{AP}, 0.1 \mathrm{M} \mathrm{CaCl}_{2}\right)$.

(although the initial release amount did not change greatly). The effect was more remarkable for CAP prepared with $0.8 \%$ AP, which produced a release rate of $0.19 \% \mathrm{~min}^{-1}$.

Figure 5 shows HC release profiles from Alg-Ca. The initial release of $\mathrm{HC}$ was minor and the drug was released gradually from Alg-Ca (CP-free). Neither alginate nor $\mathrm{CaCl}_{2}$ concentration affected drug release rate. Modification of AlgCa with $\mathrm{CP}$ also did not affect the HC release profile. HC is slightly soluble in water, and the release rate, therefore, appears to be governed by the dissolution of the drug as the solvent permeates the pores of the gel matrix.

\section{Conclusions}

In this study, CAP was prepared and used for the investigation of drug release from a drug delivery vehicle. Drug release occurs when the Alg-Ca surface comes in contact with the dissolution medium. The initial release profile of drugs contained in Alg-Ca is especially affected by the surface structure of the gel matrix. A water-soluble drug DF was immediately released from the intact Alg-Ca, and the drug release rate could be controlled by modifying the composition of the matrix with CP. However, CP and/or calcium alginate are good candidates as implanted materials for bone tissue engineering. Therefore, work is continuing to investigate the biocompatibility of CAP.

\section{Acknowledgment}

This work was supported in part by a grant from Hokuriku University (2008).

\section{References}

[1] W. J. E. M. Habraken, Z. Zhang, J. G. C. Wolke, et al., "Introduction of enzymatically degradable poly(trimethylene carbonate) microspheres into an injectable calcium phosphate cement," Biomaterials, vol. 29, no. 16, pp. 2464-2476, 2008.
[2] F. Witte, T. Calliess, and H. Windhagen, "Biodegradable synthetic implant materials,” Der Orthopäde, vol. 37, no. 2, pp. 125-130, 2008.

[3] S. C. G. Leeuwenburgh, J. A. Jansen, and G. Mikos, "Functionalization of oligo(poly(ethylene glycol)fumarate) hydrogels with finely dispersed calcium phosphate nanocrystals for bone-substituting purposes," Journal of Biomaterials Science, Polymer Edition, vol. 18, no. 12, pp. 1547-1564, 2007.

[4] W. F. Regnault, T. B. Icenogle, J. M. Antonucci, and D. Skrtic, "Amorphous calcium phosphate/urethane methacrylate resin composites. I. Physicochemical characterization," Journal of Materials Science: Materials in Medicine, vol. 19, no. 2, pp. 507515, 2008.

[5] M. Charles-Harris, S. del Valle, E. Hentges, P. Bleuet, D. Lacroix, and J. A. Planell, "Mechanical and structural characterisation of completely degradable polylactic acid/calcium phosphate glass scaffolds," Biomaterials, vol. 28, no. 30, pp. 4429-4438, 2007.

[6] M. Charles-Harris, M. A. Koch, M. Navarro, D. Lacroix, E. Engel, and J. A. Planell, "A PLA/calcium phosphate degradable composite material for bone tissue engineering: an in vitro study," Journal of Materials Science: Materials in Medicine, vol. 19, no. 4, pp. 1503-1513, 2008.

[7] C. R. Kothapalli, M. T. Shaw, J. R. Olson, and M. Wei, "Fabrication of novel calcium phosphate/poly(lactic acid) fiber composites," Journal of Biomedical Materials Research Part B, vol. 84, no. 1, pp. 89-97, 2008.

[8] E. A. Nunamaker, E. K. Purcell, and D. R. Kipke, "In vivo stability and biocompatibility of implanted calcium alginate disks," Journal of Biomedical Materials Research Part A, vol. 83, no. 4, pp. 1128-1137, 2007.

[9] M. Figliuzzi, T. Plati, R. Cornolti, et al., "Biocompatibility and function of microencapsulated pancreatic islets," Acta Biomaterialia, vol. 2, no. 2, pp. 221-227, 2006.

[10] C. G. Thanos, B. E. Bintz, and D. F. Emerich, "Stability of alginate-polyornithine microcapsules is profoundly dependent on the site of transplantation," Journal of Biomedical Materials Research Part A, vol. 81, no. 1, pp. 1-11, 2006.

[11] Y.-D. Tang, S. S. Venkatraman, F. Y. C. Boey, and L.-W. Wang, "Sustained release of hydrophobic and hydrophilic drugs from a floating dosage form," International Journal of Pharmaceutics, vol. 336, no. 1, pp. 159-165, 2007.

[12] G. R. Castro, R. R. Kamdar, B. Panilaitis, and D. L. Kaplan, "Triggered release of proteins from emulsan-alginate beads," Journal of Controlled Release, vol. 109, no. 1-3, pp. 149-157, 2005.

[13] J. Han, A.-S. Guenier, S. Salmieri, and M. Lacroix, "Alginate and chitosan functionalization for micronutrient encapsulation," Journal of Agricultural and Food Chemistry, vol. 56, no. 7, pp. 2528-2535, 2008.

[14] Y. Murata, D. Jinno, D. Liu, T. Isobe, K. Kofuji, and S. Kawashima, "The drug release profile from calcium-induced alginate gel beads coated with an alginate hydrolysate," Molecules, vol. 12, no. 11, pp. 2559-2566, 2007. 

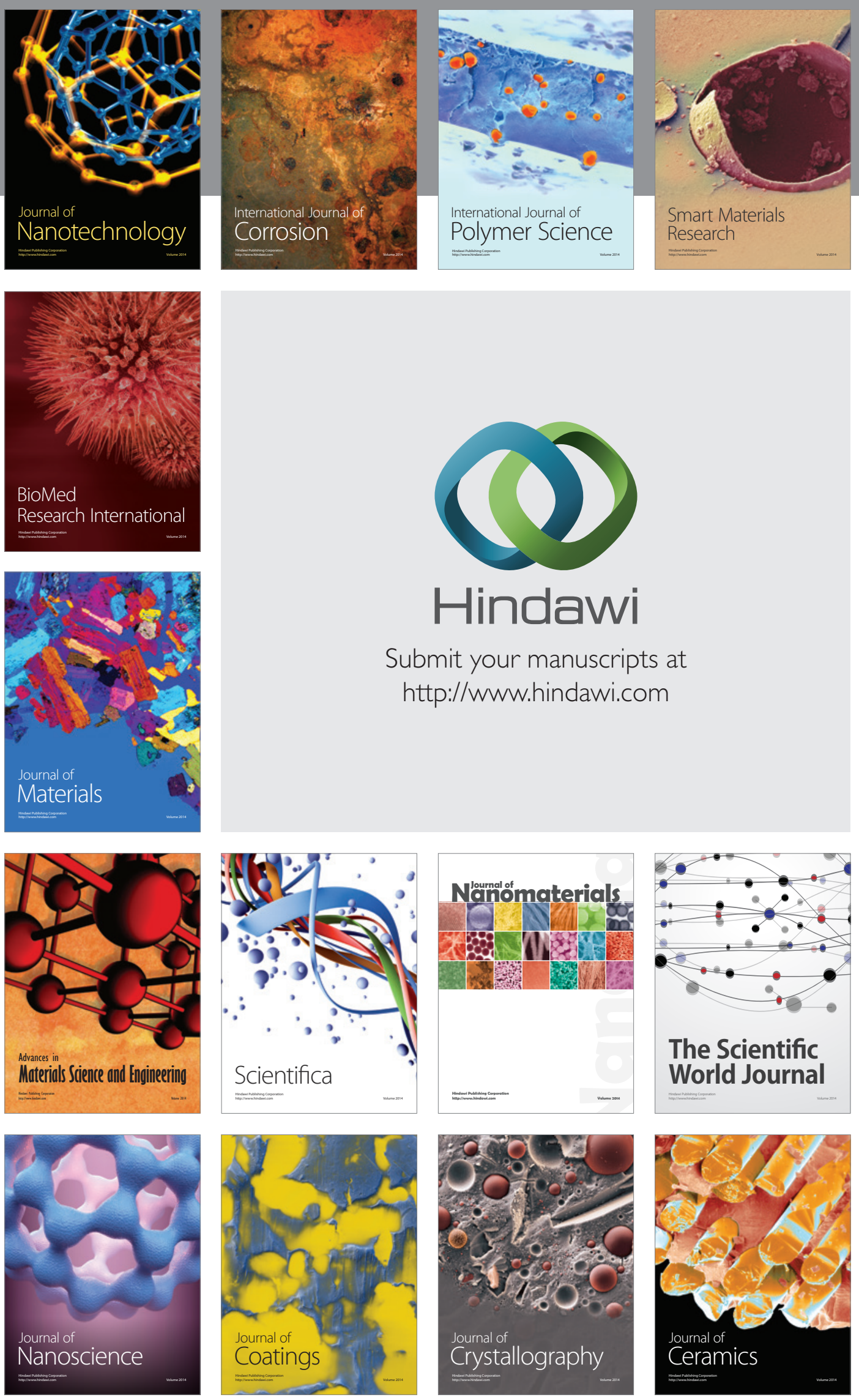

The Scientific World Journal

Submit your manuscripts at

http://www.hindawi.com

\section{World Journal}

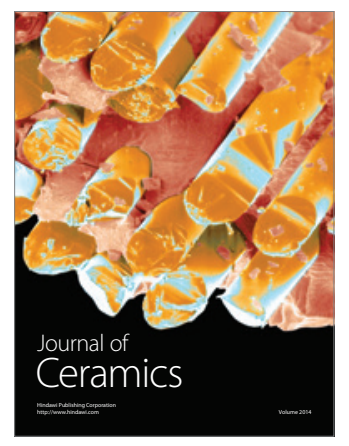

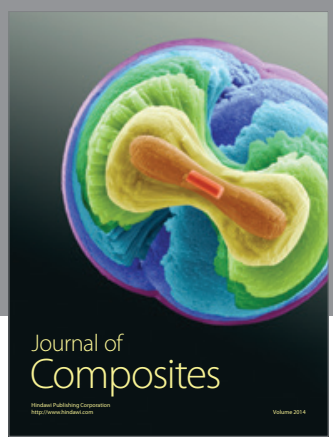
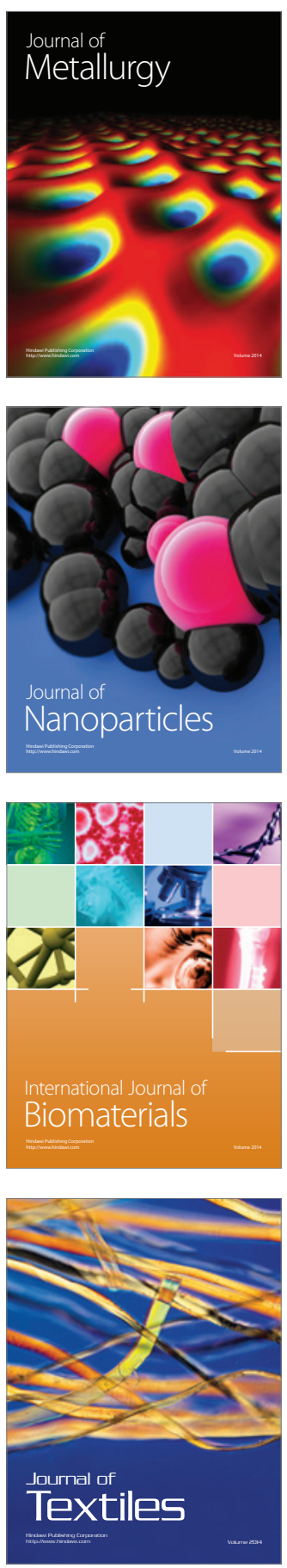